\title{
Relationship Between Hippocampal Volume, Serum BDNF, and Depression Severity Following Electroconvulsive Therapy in Late-Life Depression
}

\author{
Filip Bouckaert ${ }^{*, 1,6}$, Annemiek Dols ${ }^{2,6}$, Louise Emsell ${ }^{1,3}$, François-Laurent De Winter', Kristof Vansteelandt ${ }^{4}$, \\ Lene Claes ${ }^{3}$, Stefan Sunaert ${ }^{3}$, Max Stek ${ }^{2}$, Pascal Sienaert ${ }^{5}$ and Mathieu Vandenbulcke ${ }^{1}$ \\ 'KU Leuven, University Psychiatric Center KU Leuven, Old-age Psychiatry, Kortenberg, Belgium; ${ }^{2}$ Department of Psychiatry and the EMGO+ \\ Institute for Health and Care Research, VU University Medical Center Amsterdam, Amsterdam, The Netherlands; ${ }^{3}$ Division of Translational MRI, \\ Department of Imaging and Pathology, KU Leuven, Radiology, University Hospitals Leuven, University Psychiatric Center KU Leuven, Leuven, \\ Belgium; ${ }^{4}$ Department of Statistics, KU Leuven, University Psychiatric Center KU Leuven, Kortenberg, Belgium; ${ }^{5}$ Academic Center for ECT and \\ Neuromodulation, KU Leuven, University Psychiatric Center KU Leuven, Kortenberg, Belgium
}

Recent structural imaging studies have described hippocampal volume changes following electroconvulsive therapy (ECT). It has been proposed that serum brain-derived neurotrophic factor (sBDNF)-mediated neuroplasticity contributes critically to brain changes following antidepressant treatment. To date no studies have investigated the relationship between changes in hippocampal volume, mood, and sBDNF following ECT. Here, we combine these measurements in a longitudinal study of severe late-life unipolar depression (LLD). We treated 88 elderly patients with severe LLD twice weekly until remission (Montgomery-Åsberg Depression Rating Scale (MADRS) < I0). sBDNF and MADRS were obtained before ECT (T0), after the sixth ECT (TI), I week after the last ECT (T2), 4 weeks after the last ECT (T3), and 6 months after the last ECT (T4). Hippocampal volumes were quantified by manual segmentation of $3 T$ structural magnetic resonance images in 66 patients at T0 and T2 and in 23 patients at TO, T2, and T4. Linear mixed models (LMM) were used to examine the evolution of MADRS, sBDNF, and hippocampal volume over time. Following ECT, there was a significant decrease in MADRS scores and a significant increase in hippocampal volume. Hippocampal volume decreased back to baseline values at T4. Compared with T0, sBDNF levels remained unchanged at TI, T2, and T3. There was no coevolution between changes in MADRS scores, hippocampal volume, and sBDNF. Hippocampal volume increase following ECT is an independent neurobiological effect unrelated to sBDNF and depressive symptomatology, suggesting a complex mechanism of action of ECT in LLD.

Neuropsychopharmacology (2016) 4I, 274I-2748; doi: I 0.1038/npp.20I6.86; published online 29 June 2016

\section{INTRODUCTION}

In severe late-life depression (LLD), electroconvulsive therapy (ECT) is the most effective treatment available and the safest choice for those who cannot tolerate or respond poorly to medications (Kerner and Prudic, 2014). ECT-induced structural brain changes have been documented in both animal and human studies, but the cause and extent of these structural changes as well as the relationship with therapeutic efficacy remains unclear (Bouckaert et al, 2014). To date, hippocampal volume changes and neuroplasticity-related mechanisms (neurogenesis, synaptogenesis, angiogenesis, or gliogenesis) have been studied most extensively (Bouckaert et al, 2014).

\footnotetext{
*Correspondence: Dr F Bouckaert, KU Leuven, University Psychiatric Center KU Leuven, Old-age Psychiatry, Leuvensesteenweg 517 , Kortenberg 3070, Belgium, Tel: 00322758 0985, Fax: 0032275953 80,

E-mail: Filip.Bouckaert@upckuleuven.be

${ }^{6}$ Filip Bouckaert \& Annemiek Dols share first authorship.

Received I March 2016; revised I5 May 20I5; accepted 2 June 2016; accepted article preview online 8 June 2016
}

Although major depression is not generally considered to be a hippocampal disorder, both biochemical and structural abnormalities of the hippocampus are frequently associated with the illness (Pilar-Cuellar et al, 2013). Moreover, the hippocampus and brain regions that mediate emotional and cognitive regulation are anatomically connected, lending support to the role of the hippocampus in depressionrelated circuitry (Price and Drevets, 2010; Abbott et al, 2014). Several recent magnetic resonance imaging (MRI) studies have described hippocampal volume changes following ECT (Nordanskog et al, 2010; Tendolkar et al, 2013; Abbott et al, 2014; Dukart et al, 2014; Nordanskog et al, 2014; Joshi et al, 2015; Jorgensen et al, 2015; Ota et al, 2015; Bouckaert et al, 2015; Sartorius et al, 2016). Some of these studies have also addressed structural changes in other brain areas relevant to depressive disorders, such as dorsolateral prefrontal cortex, orbitofrontal cortex (Jorgensen et al, 2015), lateral temporal cortex (Bouckaert et al, 2015), caudate nucleus (Bouckaert et al, 2015), and amygdala (Tendolkar et al, 2013; Jorgensen et al, 2015; Joshi et al, 2015; Ota et al, 
2015; Sartorius et al, 2016). Only one study did not detect gray matter volume changes (Nickl-Jockschat et al, 2016).

Brain-derived neurotrophic factor (BDNF)-mediated neuroplasticity may contribute critically to brain changes following antidepressant treatment (Vaidya et al, 1999; Molendijk et al, 2013). Similar to pharmacological interventions, peripheral BDNF levels increase after ECT in some but not all studies (Polyakova et al, 2015). Moreover, there is strong evidence that decreased BDNF is associated with age-related hippocampal dysfunction and increased risk for depression (Erickson et al, 2010; Erickson et al, 2012).

The 'anatomical-ictal hypothesis' (Bolwig, 2011) aims to unify the concepts introduced above by relating hippocampal dysfunction and neuroplasticity to the working mechanism of ECT. It proposes that seizure activity in the limbic system induces neurotrophic effects crucial for therapeutic efficacy (Bolwig, 2011). This hypothesis is in line with the preclinically developed notion that stress is associated with neuronal atrophy and reduced neurogenesis (Duman and Aghajanian, 2012), impaired synaptic plasticity, and dendritic arborization in the hippocampus (Sousa et al, 2000), whereas antidepressants and electroconvulsive stimulation (ECS; the animal model of ECT) stimulate neurogenesis (Bolwig, 2011), synaptogenesis, gliogenesis, and angiogenesis (Duman and Aghajanian, 2012; Bouckaert et al, 2014).

To date, no studies have examined the association between hippocampal volume, therapeutic efficacy, and serum BDNF (sBDNF) following ECT in patients with LLD. Here, we combined these variables in a longitudinal study of severe late-life unipolar depression in order to validate the combined anatomical-ictal theory.

We hypothesized that, given the decrease in both sBDNF and hippocampal volume in depression (Bus et al, 2012; Erickson et al, 2012; Sexton et al, 2013), ECT would increase the level of sBDNF, which would be associated with an increase in hippocampal volume, and subsequent positive treatment response.

\section{MATERIALS AND METHODS}

\section{Subjects and Characteristics}

Subjects were included at the University Psychiatric Center KU Leuven, Belgium, and GGZinGeest, Amsterdam, the Netherlands. Data collection started in January 2011 and was finished in July 2014. The Leuven cohort included 28 patients whose data were used in a previous voxel-based analysis investigating gray matter changes 1 week after the completion of ECT (Bouckaert et al, 2015). All subjects were 55 years and older (LLD) and had a DSM-IV-defined major depressive disorder, with or without psychotic symptoms. Age at first depressive episode before 55 years was classified as early-onset depression (EOD), a first episode at 55 years and older was defined as late-onset depression (LOD). The diagnoses were made by a psychiatrist and confirmed by the Mini International Neuropsychiatric Interview (Sheehan et al, 1998). Exclusion criteria were another major psychiatric illness, a major neurological illness (including Parkinson's disease, stroke, and dementia), and contraindications to MRI. Psychotropic medication was discontinued at least 1 week before ECT, or, if deemed impossible, kept stable from 6 weeks before ECT and during the ECT course.
Trazodone ( $\leqslant 100 \mathrm{mg})$, clotiapine $(\leqslant 40 \mathrm{mg})$, or lorazepam $(\leqslant 5 \mathrm{mg})$ was allowed pro re nata (agitation, anxiety, and insomnia). After T2 (1 week post ECT), further treatment was based on the clinical judgment of the treating psychiatrist: $9.1 \%(n=8)$ received an SSRI, $65.9 \%(n=58)$ nortriptyline, $30.7 \% \quad(n=27)$ a combination of nortriptyline and lithium, $19.48 \% \quad(n=15)$ antidepressants combined with continuation ECT (cECT), and $3.90 \% \quad(n=3)$ received cECT only.

All patients provided written informed consent. The ethical committees of the two centers approved this study, which was conducted according to the declaration of Helsinki.

\section{Study Design}

Subjects were assessed 1 week before ECT (T0), after the sixth ECT (T1), 1 week (T2), 4 weeks (T3) after the last ECT, and also 6 months after the last ECT (T4).

\section{ECT Procedure}

ECT was administered twice a week with a constant-current brief-pulse device (Thymatron System IV, Somatics, IL, USA). Anesthesia was achieved with intravenous administration of etomidate $(0.2 \mathrm{mg} / \mathrm{kg})$ and succinylcholine $(1 \mathrm{mg} / \mathrm{kg})$. Motor and electroencephalographic seizures were monitored to ensure adequate duration and quality. Subjects were all treated with right unilateral (RUL) ECT with stimulus intensity six times the initial seizure threshold (ST), as determined by empirical dose titration at the first treatment, until remission. Subjects who failed to respond RUL ECT after the sixth treatment were switched to bitemporal ECT $(1.5 \times$ ST; Dutch Guideline for ECT, 2010). All patients were treated with brief pulse $(0.5-1.0 \mathrm{~ms})$ ECT.

\section{Clinical Instruments}

Depressive symptoms were monitored at time points T0, T1, T2, and T3, using the Montgomery-Åsberg Depression Rating Scale (MADRS; Montgomery and Asberg, 1979). Response was defined as a decrease in MADRS scores of at least $50 \%$, remission as MADRS score $<10$ at two consecutive weekly assessments, and relapse as a MADRS score $>15$, a re-admission, restart of ECT, or suicide.

\section{BDNF Protein Measurements}

Blood samples were taken between between 0730 and 0930 hours after an overnight fast. Serum was immediately separated and stored at $-85^{\circ} \mathrm{C}$ for a period varying between 7 and 43 months until being assayed. BDNF protein levels were measured using the Emax Immuno Assay System from Promega according to the manufacturer's protocol (Madison, WI, USA) in one laboratory (Maastricht University). Undiluted serum was acid-treated as this reliably increased the detectable BDNF in a dilution-dependent manner. Greiner Bio-One high-affinity 96-well plates were used. Serum samples were diluted 100 times, and the absorbency was read in duplicate using a Bio-Rad (Hercules, CA, USA) Benchmark microplate reader at $450 \mathrm{~nm}$. The intra- and inter-assay coefficients of variation were found to be within $3 \%$ and $14 \%$, respectively. No subjects had BDNF values below the 
reliable detection threshold of $1.56 \mathrm{ng} / \mathrm{ml}$. These values were set at the lower detection limit. Positive outliers were trimmed to the mean $+3 \mathrm{SD}$ value. This procedure was performed at four time points: T0, T1, T2, and T3.

\section{MRI Acquisition and Processing}

Structural MRI was performed at T0 $(n=66)$, T2 $(n=66)$, and T4 $(n=23)$. High-resolution $3 \mathrm{D}$ T1-weighted images were acquired using an eight-channel head-coil with a $3 \mathrm{D}$ turbo field echo sequence on a 3T Philips Intera scanner in Leuven and on a $3 \mathrm{~T}$ GE Signa HDxt scanner in Amsterdam $\left(\mathrm{TR}=9.6 \mathrm{~s}, \mathrm{TE}=4.6 \mathrm{~s}\right.$, flip angle $=8^{\circ}$, slice thickness $=1.2 \mathrm{~mm}$, in-plane voxel size $=0.98 \times 0.98 \times 1.2 \mathrm{~mm}^{3}, 182$ slices, acquisition time $=383 \mathrm{~s}$ ).

\section{Hippocampal Volume Measurements}

A single-trained rater blinded to time point manually delineated the hippocampus in native space following an initial automatic segmentation step (van der Lijn et al, 2008). Manual editing was performed using ITK-SNAP version 2.4 (http://www.itksnap.org/pmwiki/pmwiki.php) in accordance with the HarP guidelines (Frisoni et al, 2015). Hippocampal volumes were normalized using the following equation (Jack Jr. et al, 1989): normalized hippocampal volume = original hippocampal volume $(\mathrm{OHV})$ - linear regression coefficient $\times$ (total intracranial volume (TIV) - mean total intracranial volume). The coefficient was derived from a linear regression of TIV and $\mathrm{OHV}$. TIV was obtained from an automated segmentation of gray matter, white matter, and cerebrospinal fluid (Jain et al, 2015). Intra-rater reliability was determined using randomly selected scans segmented at two time points at least 1 month apart. The intraclass correlation coefficient (Cronbach's $\alpha$ ) was 0.96 for the left hippocampus and 0.95 for the right hippocampus.

\section{Statistical Analysis}

Baseline associations. To assess the association between illness severity (as measured by MADRS), sBDNF, and total hippocampal volume at baseline, Pearson's correlations were used. To determine whether LOD and age were related to these three variables of interest at baseline (T0), we performed multiple regression analyses adjusted for site (Amsterdam versus Leuven) and medication use (yes or no).

Longitudinal changes. To examine the evolution of MADRS, sBDNF, and hippocampal volume over the different time points (T0, T1, T2, T3, and T4), multilevel or linear mixed models (LMM) were estimated with repeated measurements (level 1) being nested within patients (level 2). Moreover, for each of the variables of interest-MADRS, sBDNF, and hippocampal volume-the effects of LOD and age were examined and all analyses were adjusted for site, medication use during ECT, switching from unilateral to bitemporal electrode position (yes or no), and the total number of received ECT sessions. Model selection was based on likelihood ratio tests and information criteria. When the analyses revealed significant differences between time points (T0-T4), pair-wise post hoc comparisons between time points were performed using Tukey-Kramer adjustments.
Coevolution of MADRS, sBDNF, and hippocampal volume over time. To examine the coevolution of MADRS, sBDNF, and hippocampal volume over time, a multivariate LMM for change was used to investigate whether participants showed the same rate of change on the three different variables. The best fitting model for each of the variables was selected based on graphical inspection of the data, likelihood ratio tests, and information criteria. To investigate the coevolution or rate of change of the different variables, the model not only yields estimates of fixed effects, random variance parameters, random covariance parameters, and error terms for each outcome variable separately, but also random covariances for parameters representing different outcome variables. For example, a significant covariance between the random subject-specific slopes for time for MADRS on the one hand, and the random subject-specific slopes for time for sBDNF on the other hand would indicate that subjects are characterized by the same linear evolution over time on both outcome variables. All analyses were performed in SAS 9.1 (SAS Institute, Cary, NC).

Missing data. With respect to missing data, we used the direct likelihood approach (also called likelihood-based ignorable analysis; Beunckens et al, 2005). The direct likelihood approach makes use of all available information without the need either to delete or to impute measurements, and makes appropriate adjustments-valid under the assumption of missing at random-to parameters at times when data are incomplete because of the within-patient correlation.

\section{RESULTS}

\section{Clinical Characteristics}

We included 88 subjects ( $34 \%$ males) with a mean age of 72.6 (range 55-92; $\mathrm{SD}=8.5$ ). Age at onset of first depression was late ( $>55$ years) in $54.5 \%$ of the subjects. The mean duration of illness (in months) was 12.44 (range 1-144; SD =20.12). The mean number of depressive episodes was 2.92 (range $0-20$; SD =3.5). The mean administered number of ECT sessions was 12 (range $4-36 ; \mathrm{SD}=5.6$ ). In 73 subjects $(83 \%)$, medication was discontinued before ECT. Fifteen subjects $(17 \%)$ were kept on a stable dose of nortriptyline, venlafaxine, sertraline, or escitalopram. Twenty-seven subjects $(30.7 \%)$ who failed to respond initially to RUL ECT were switched after the sixth treatment to bitemporal ECT.

\section{Baseline Correlations of Illness Severity, sBDNF, and Hippocampal Volume}

Baseline sBDNF correlated significantly with baseline right hippocampal volume $(r=0.27 ; p=0.01)$, left hippocampal volume $(r=0.30 ; p=0.008)$, and total hippocampal volume $(r=0.30 ; p=0.007$; Figure 1$)$, but not with illness severity. There were also no significant correlations between hippocampal volume and illness severity. In addition, we examined whether LOD and age were related to MADRS, sBDNF, and total hippocampal volume at baseline adjusting for site and medication use. Multiple regression revealed that LOD, $t(1)=-0.19, p=0.85$, and age, $t(1)=-0.32, p=0.75$, were not related to MADRS at baseline. Both variables were 
also not related to sBDNF at baseline, LOD, $t(1)=0.52$, $p=0.61$, and age, $t(1)=-0.66, p=0.51$. Finally, LOD was not related to total hippocampal volume at baseline, $t(1)=-0.57$, $p=0.57$, but age was, $t(1)=-2.82, p=0.006$, with older patients having a smaller total hippocampal volume. Separate analyses for left and right hippocampal volume revealed similar results.

\section{Evolution of Clinical Response}

The LMM for the MADRS revealed significant differences between the different time points, $F(4,278)=94.67$, $p<0.0001$. Pairwise comparisons (see Table 1) among the different time points revealed significant differences between

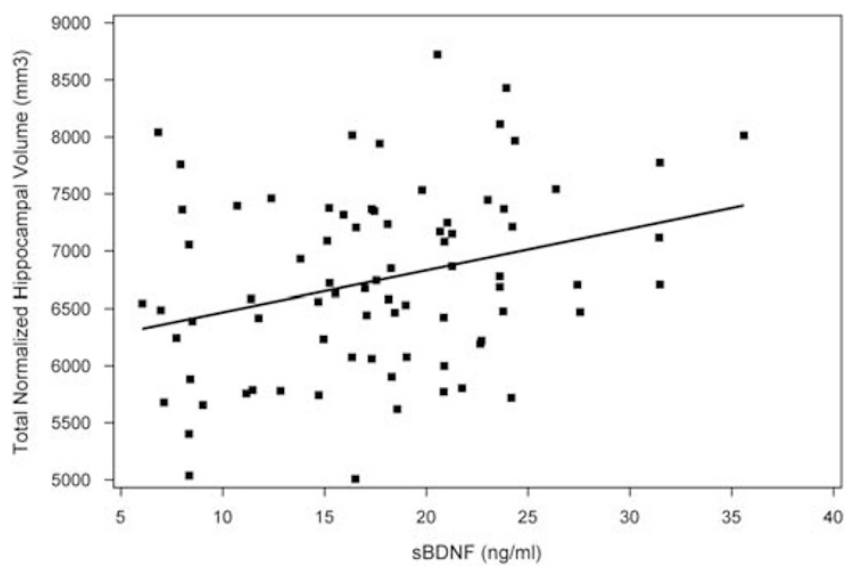

Figure I Serum brain-derived neurotrophic factor (sBDNF) at baseline positively correlates with normalized total hippocampal volume at baseline. the MADRS both at baseline (T0) and all other time points, as well as after the sixth ECT (T1) and all other time points. There were no significant differences between MADRS scores 1 week post ECT (T2), 4 weeks post ECT (T3), and 6 months post ECT (T4) (Table 1 and Figure 2). Response, remission, and relapse data are included in Table 1.

\section{Evolution of sBDNF}

The LMM for sBDNF revealed no significant differences in sBDNF over the different time points, $F(3,222)=0.81$, $p=0.50$ (Table 1 and Figure 3 ). In addition, there were no significant effects of LOD, $\mathrm{F}(1,222)=0.19, p=0.60$, or age, $\mathrm{F}(1,222)=2.82, p=0.60$.

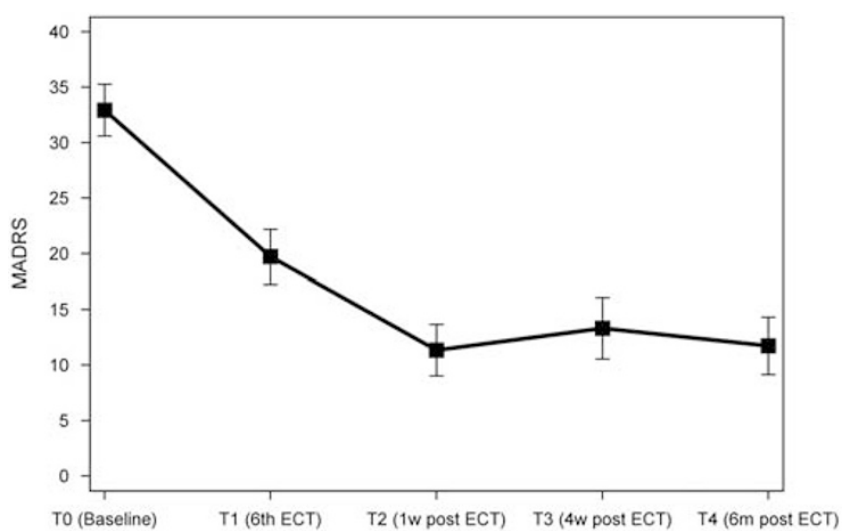

Figure 2 Evolution of Montgomery-Åsberg Depression Rating Scale (MADRS) at different time points.

Table I Clinical Results, sBDNF Values, and Hippocampal Volume of the Study Sample at Each Time Point

\begin{tabular}{|c|c|c|c|c|c|}
\hline & T0 (baseline) & TI (6th ECT) & T2 (I w post ECT) & T3 (4w post ECT) & T4 (6m post ECT) \\
\hline MADRS score mean (SE; $n$ ) & $32.93^{\mathrm{a}}(1.18 ; 85)$ & $19.72^{b}(1.25 ; 72)$ & $11.36^{c}(1.18 ; 85)$ & $13.33^{\mathrm{C}}(1.41 ; 61)$ & $11.73^{c}(1.31 ; 69)$ \\
\hline Change in MADRS since baseline (SE) & 0 & $-13.21(1.34)$ & $-21.57(1.28)$ & $-19.6 \mid(1.48)$ & $-21.20(1.39)$ \\
\hline Response $n(\%)$ & & $35(49.30)$ & $68(80)$ & 45 (73.77) & $52(75.36)$ \\
\hline Remission n (\%) & & $13(|8.3|)$ & $58(68.24)$ & $29(47.54)$ & $4 \mid(59.42)$ \\
\hline Relapse $n(\%)$ & & & & $20(23.53)$ & $36(42.35)$ \\
\hline Change in sBDNF since baseline (SE) & 0 & $0.26(0.61)$ & $0.20(0.59)$ & $0.97(0.65)$ & \\
\hline Left hippocampal volume $\left(\mathrm{mm}^{3}\right)$ mean (SE; $n$ ) & $3351.65^{\mathrm{a}}(65.93 ; 79)$ & & $3418.35^{b}(66.60 ; 66)$ & & $3293.21^{\mathrm{a}}(73.63 ; 23)$ \\
\hline $\begin{array}{l}\text { Change in left hippocampal volume }\left(\mathrm{mm}^{3}\right) \\
\text { since baseline }\end{array}$ & 0 & & $66.70(27.72)$ & & $-58.43(41.29)$ \\
\hline Right hippocampal volume $\left(\mathrm{mm}^{3}\right)$ mean (SE; $n$ ) & $3419.85^{\mathrm{a}}(65.93 ; 79)$ & & $3539.88^{b}(66.60 ; 66)$ & & $3447.52^{\mathrm{a}}(73.63 ; 23)$ \\
\hline $\begin{array}{l}\text { Change in right hippocampal volume }\left(\mathrm{mm}^{3}\right) \\
\text { since baseline }\end{array}$ & 0 & & $120.03(27.72)$ & & $27.67(41.29)$ \\
\hline
\end{tabular}

MADRS score, Montgomery-Åsberg Depression rating scale score; $n$, number of subject; sBDNF, serum brain-derived neurotrophic factor; T0, baseline; TI, after the sixth ECT; T2, I week after last ECT; T3, 4 weeks after last ECT; T4, 6 months after last ECT.

Response defined as decrease in MADRS scores of at least 50\%; remission defined as MADRS score $<10$ at two consecutive weekly assessments with a week interval; relapse defined as re-admission, restart of ECT, suicide, or a MADRS score $>15$.

All analyses are adjusted for site, medication use during ECT, switching from unilateral to bitemporal electrode position, and number of ECT received. Means that do not share the same superscript $\mathrm{a}$, b or $\mathrm{c}$ are significantly different $(p<0.05)$ using Tukey-Kramer adjustment for multiple comparisons. 


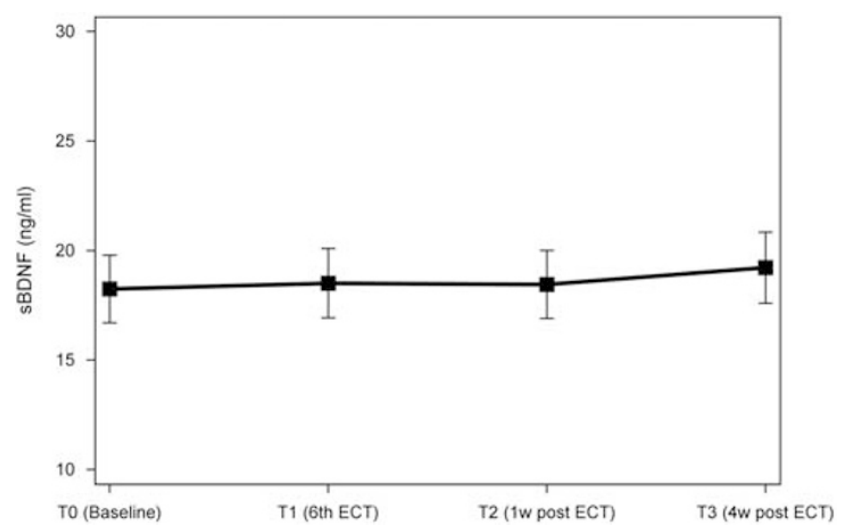

Figure 3 Evolution of serum brain-derived neurotrophic factor (sBDNF) at different time points.

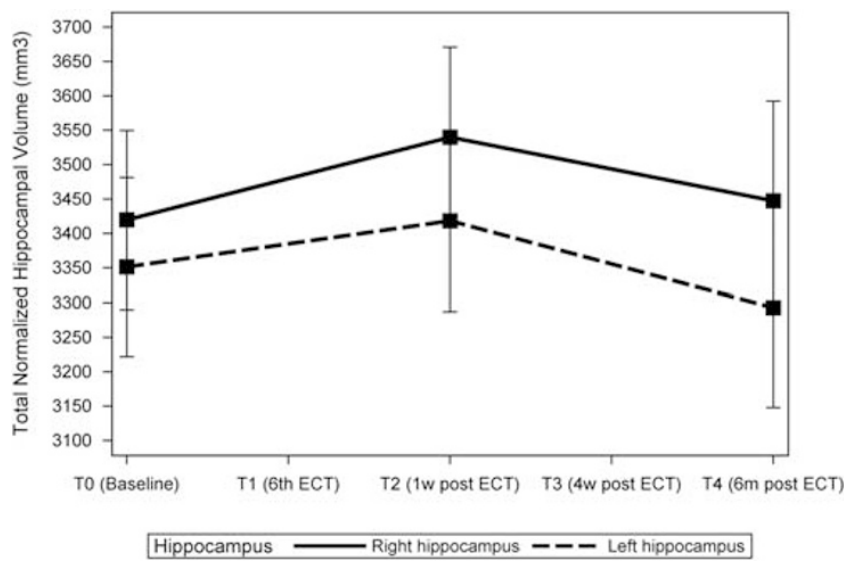

Figure 4 Evolution of hippocampal volume at different time points.

\section{Evolution of Hippocampal volume}

The LMM for hippocampal volume revealed significant differences between time points, $\mathrm{F}(2,252)=13.03, p<0.0001$ (Table 1 and Figure 4). Pairwise post hoc comparisons (see Table 1) among these time points showed that hippocampal volume was significantly lower at baseline (T0) compared with 1 week post ECT (T2) but not significantly different from hippocampal volume 6 months after ECT (T4). Hippocampal volume was also significantly different at T2 and T4. In addition, hippocampal volume was significantly lower in the left hemisphere (difference $=154.30 \mathrm{~mm}^{3} \quad(\mathrm{SE}=48.07)$, $\mathrm{F}(1,252)=31.31, p<0.0001)$, but the evolution in hippocampal volume over the different time points was similar for left and right hippocampi, $\mathrm{F}(2,252)=1.70, p=0.19$. Finally, there was no effect of LOD, $\mathrm{F}(1,252)=0.93, p=0.034$, but at all time points older patients tend to have a smaller hippocampal volume, $\mathrm{F}(1,256)=6.94, p=0.0090$. Analyses for the total hippocampal volume yielded similar results.

\section{Coevolution between Hippocampal Volume, sBDNF, and Mood}

Estimation of the multivariate mixed model for change indicated no evidence for a coevolution between hippocampal volume, sBDNF, and depression severity as indicated by the lack of significant (co)variances in the slope parameters of these variables.

\section{DISCUSSION}

To our knowledge, this is the first study investigating the relationship between hippocampal volume, depressive symptomatology and sBDNF following ECT in LLD. As expected, depressive symptomatology improved significantly one week after the last ECT. Hippocampal volume initially increased and then normalized 6 months after the last ECT. sBDNF did not change significantly over the assessment period. We did not find any coevolution between changes in MADRS scores, hippocampal volume and sBDNF.

\section{Relationship between Clinical Response and sBDNF}

Our results are in line with reports showing no changes in sBDNF during and after ECT, as well as studies showing no correlation with depression improvement (Jorgensen et al, 2015; Rapinesi et al, 2015). Although BDNF can have a crucial role in the development of depression and its recovery (Molendijk et al, 2013; Bus et al, 2012, 2015), our study seems to indicate that improvements in depression and sBDNF are unrelated. The inability to elevate sBDNF levels following ECT could be because of an age-related less responsive BDNF system (Calabrese et al, 2013; Rapinesi et $a l, 2015)$. This could well be related to an age-related decrease in sBDNF levels. In our sample, the mean sBDNF level was $18.27 \mathrm{ng} / \mathrm{ml}$, which is in line with earlier reports of elderly populations (Erickson et al, 2010; Bus et al, 2012; Rapinesi et al, 2015) but lower compared with levels ranging from 9.5 to $29.0 \mu \mathrm{g} / \mathrm{ml}$ in non-elderly depressed patients (Sen et al, 2008). Differences in the sBDNF level could also be explained by sample characteristics and protocols used (eg, urbanicity, sex, smoking status, time of blood withdrawal, and so on; Bus et al, 2011). Similar to the observation from a large longitudinal study showing that initiation or discontinuation of antidepressants is not associated with sBDNF change ( $p=0.72$; Bus et al, 2015), we did not find any differences between the subjects who continued antidepressant therapy with the non-medicated sample.

\section{Relationship between Depressive Symptomatology and Hippocampal Volume Changes}

Although hippocampal volume increased after ECT, and depressive symptoms decreased, we failed to find a relationship between these variables. This is in line with previous studies (Nordanskog et al, 2010; Tendolkar et al, 2013; Abbott et al, 2014; Dukart et al, 2014; Nordanskog et al, 2014; Bouckaert et al, 2015; Jorgensen et al, 2015; Ota et al, 2015; Sartorius et al, 2016). Only two studies found an association between hippocampal volume increase and therapeutic efficacy (Dukart et al, 2014; Joshi et al, 2015). Our data suggest that the hippocampal volume increase after ECT is transient and reverts to baseline within 6 months, even though the antidepressant effect is sustained. Similar transient changes unrelated to clinical changes have recently been reported with hippocampal volume increase 1 week (Nordanskog et al, 2014; Jorgensen et al, 2015) and volume 
decrease 4 weeks (Jorgensen et al, 2015) and 6 months (Nordanskog et al, 2014) after the last ECT.

\section{Relationship between Hippocampal Volume Changes and SBDNF Changes}

At baseline, higher sBDNF levels were associated with larger hippocampi, a result that was also shown by Erickson et al (2010) in 142 older adults without dementia and without depression.

In preclinical research, BDNF has been convincingly demonstrated to relate to synaptic plasticity and neurogenesis in the hippocampal dentate gyrus (Erickson et al, 2010). In addition, ECS increases BDNF mRNA and protein concentration in the rodent brain, with largest effect sizes measured in the dentate gyrus (Polyakova et al, 2015). Unlike these preclinical data, we were not able to show a relationship between changes in hippocampal volume and sBDNF. Although several studies have reported positive correlations between sBDNF and BDNF in both the prefrontal cortex and the hippocampus, suggesting a link between peripheral and central sources of BDNF (Sartorius et al, 2009; Elfving and Wegener, 2012), the degree to which sBDNF reflects BDNF levels in some brain areas remains a matter of speculation (Erickson et al, 2012). A delayed increase of sBDNF at 2 or 3 weeks after the last ECT has been described recently (Bumb et al, 2015), suggesting that the sBDNF peak could have been missed in our study or may not coincide with hippocampal volume changes.

\section{Time Course of ECT-Induced Hippocampal Volume Changes}

Preclinical studies suggest ECS-related structural plasticity (neurogenesis, synaptogenesis, angiogenesis, or gliogenesis; Bouckaert et al, 2014). However, given the low number of new neurogenic cells produced in adulthood (Spalding et al, 2013), dendritic remodeling, synapse turnover, increased glial cell, and capillary density rather than neuronal replacement may account for ECT-induced volumetric changes (Bessa et al, 2009). Accordingly, Nordgren et al (2013) hypothesized that ECT causes transient downregulation of the key molecules needed to stabilize synaptic structures and to prevent calcium influx, and a simultaneous increase in neurotrophic factors, thus providing a short window of increased structural synaptic plasticity. Their findings indicate that the window of plasticity with increased neuromodulation is followed by a later phase during which the synaptic network is stabilized in a new, slightly altered configuration. The structural synaptic rearrangements in this altered configuration could conceivably be causing the volume decrease at T4 following the initial increase at T2. These findings are in line with the partial reversion of ECSinduced synaptic remodeling following 12 weeks of recovery seen in rats (Bolwig and Jorgensen, 1980). Interestingly, 2 of the 23 patients who had a third MRI received cECT (with antidepressants and no lithium) after T2 and showed no volume decrease at T4 (data not shown). Although speculative, this suggests no reversion of the ECT-induced structural changes when ECT is continued.

\section{Alternative Processes Accounting for Hippocampal Volume Changes following ECT}

Other than neuro-, synapto-, glio-, and vasculogenesis, processes such as brain tissue reaction to the electric current, possibly associated with edema, blood-brain barrier breach, and activity level should be considered as alternative explanations for hippocampal volume change.

In a previous study using voxel-based morphometry, we described a marked lateralization of gray matter volume increase toward the stimulation side, suggesting that a current-related effect may contribute to hippocampal volume changes (Bouckaert et al, 2015). In our current study, however, there was no significant difference in evolution of hippocampal volume between the left and right hippocampus over the different time points, and there was no difference in the degree of volume change between the patients who were treated with RUL ECT and those who were switched to bitemporal ECT.

Although early preclinical research has demonstrated that postictal dendritic swelling could be reversible (Hesse and Teyler, 1976), and the transient intra-ictal breach in the blood-brain barrier (Andrade and Bolwig, 2014) might facilitate local swelling, there is evidence suggesting that edema is unlikely to be the primary cause of the observed volume changes (Kunigiri et al, 2007; Szabo et al, 2007; Nordanskog et al, 2010; Jorgensen et al, 2015).

As hippocampal growth due to exercise has been described (Erickson et al, 2012), normalization of activity-level post ECT should be investigated as a covariate influencing hippocampal volume increase.

To the best of our knowledge, this was the first study to investigate the relationship between hippocampal volume, depressive symptom severity, and sBDNF following ECT in LLD. It was also the largest study to investigate the coevolution of these key parameters in patients with unipolar depression following ECT, and used the gold-standard manual segmentation approach for hippocampal volume measurement. Some limitations should be addressed. First, a limited number of subjects were not medication-free. As antidepressants are likely to influence sBDNF and gray matter volume (Frodl et al, 2008; Sen et al, 2008), this could have influenced our results. The subjects who continued pharmacotherapy had been taking these drugs for at least 6 weeks. We did not, however, find any significant differences in the evolution of sBDNF, hippocampal volume, or MADRS between the medicated and non-medicated participants. Second, as there were only 23 subjects of the 69 at 6-month follow-up who had an MRI, we should be cautious about the interpretation and conclusions at 6 months post-ECT. Although the direct likelihood approach is recommended to deal with missing data (Beunckens et al, 2005), we also performed an auxiliary Complete Case (CC) analysis $(n=22)$ for MADRS, sBDNF, and hippocampal volume (data not shown), which yielded similar results. Third, the absence of matched comparison subjects did not allow us to control for variance induced by repeated measurements. However, the longitudinal design of the study permitted powerful within-subject analyses and the participants served as their own control for identifying volumetric changes specifically from ECT. Fourth, although we assume that the hippocampus increases in volume 
physically, the measurements are based on MRI signal intensity. Cousins et al (2013) demonstrated that lithiuminduced GM volume increase detected by VBM may be exaggerated by alterations in the $\mathrm{T} 1$ properties of the tissue of interest (Cousins et al, 2013). Whereas these effects are likely to be more pronounced in automated morphometry methods, it remains possible that we are not detecting a physical volume increase but localizing the effect of ECTinduced tissue change.

In conclusion, ECT induces a significant but transient hippocampal volume increase. Contrary to the combined anatomical-ictal hypothesis, our data suggest that hippocampal volume increase following ECT is an independent neurobiological effect that is unrelated to sBDNF and depressive symptomatology, suggesting a complex mechanism of action of ECT in LLD.

\section{FUNDING AND DISCLOSURE}

MV was supported by the Research Foundation-Flanders (Fonds Wetenschappelijk Onderzoek) Project G.0746.09. The authors declare no conflict of interest.

\section{ACKNOWLEDGMENTS}

We thank Saurabh Jain (icoMetrix, Belgium), ResPECT (Research consortium for Psychiatry and ECT), and Anna Paauw (data management, GGZinGeest, the Netherlands).

\section{REFERENCES}

Abbott CC, Jones T, Lemke NT, Gallegos P, McClintock SM, Mayer AR et al (2014). Hippocampal structural and functional changes associated with electroconvulsive therapy response. Transl Psychiatry 4: e483.

Andrade C, Bolwig TG (2014). Electroconvulsive therapy, hypertensive surge, blood-brain barrier breach, and amnesia: exploring the evidence for a connection. J ECT 30: 160-164.

Bessa JM, Ferreira D, Melo I, Marques F, Cerqueira JJ, Palha JA et al (2009). The mood-improving actions of antidepressants do not depend on neurogenesis but are associated with neuronal remodeling. Mol Psychiatry 14: 739.

Beunckens C, Molenberghs G, Kenward MG (2005). Direct likelihood analysis versus simple forms of imputation for missing data in randomized clinical trials. Clin Trials 2: 379-386.

Bolwig TG (2011). How does electroconvulsive therapy work? Theories on its mechanism. Can J Psychiatry 56: 13-18.

Bolwig TG, Jorgensen OS (1980). Synaptic proteins after electroconvulsive stimulation: reversibility and regional differences in the brain. Acta Psychiatr Scand 62: 486-493.

Bouckaert F, De Winter FL, Emsell L, Dols A, Rhebergen D, Wampers $\mathrm{M}$ et al (2015). Grey matter volume increase following electroconvulsive therapy in patients with late life depression: a longitudinal MRI study. J Psychiatry Neurosci 40: 140322.

Bouckaert F, Sienaert P, Obbels J, Dols A, Vandenbulcke M, Stek M et al (2014). ECT: its brain enabling effects: a review of electroconvulsive therapy-induced structural brain plasticity. J ECT 30: 143-151.

Bumb JM, Aksay SS, Janke C, Kranaster L, Geisel O, Gass P et al (2015). Focus on ECT seizure quality: serum BDNF as a peripheral biomarker in depressed patients. Eur Arch Psychiatry Clin Neurosci 265: 227-232.
Bus BA, Molendijk ML, Penninx BJ, Buitelaar JK, Kenis G, Prickaerts J et al (2011). Determinants of serum brain-derived neurotrophic factor. Psychoneuroendocrinology 36: 228-239.

Bus BA, Molendijk ML, Tendolkar I, Penninx BW, Prickaerts J, Elzinga BM et al (2015). Chronic depression is associated with a pronounced decrease in serum brain-derived neurotrophic factor over time. Mol Psychiatry 20: 602-608.

Bus BA, Tendolkar I, Franke B, de Graaf J, den Heijer M, Buitelaar JK et al (2012). Serum brain-derived neurotrophic factor: determinants and relationship with depressive symptoms in a community population of middle-aged and elderly people. World J Biol Psychiatry 13: 39-47.

Calabrese F, Guidotti G, Racagni G, Riva MA (2013). Reduced neuroplasticity in aged rats: a role for the neurotrophin brainderived neurotrophic factor. Neurobiol Aging 34: 2768-2776.

Cousins DA, Aribisala B, Nicol Ferrier I, Blamire AM (2013). Lithium, gray matter, and magnetic resonance imaging signal. Biol Psychiatry 73: 652-657.

Dukart J, Regen F, Kherif F, Colla M, Bajbouj M, Heuser I et al (2014). Electroconvulsive therapy-induced brain plasticity determines therapeutic outcome in mood disorders. Proc Natl Acad Sci USA 111: 1156-1161.

Duman RS, Aghajanian GK (2012). Synaptic dysfunction in depression: potential therapeutic targets. Science 338: 68-72.

Elfving B, Wegener G (2012). Electroconvulsive seizures stimulate the vegf pathway via mTORC1. Synapse 66: 340-345.

Erickson KI, Miller DL, Roecklein KA (2012). The aging hippocampus: interactions between exercise, depression, and BDNF. Neuroscientist 18: 82-97.

Erickson KI, Prakash RS, Voss MW, Chaddock L, Heo S, McLaren M et al (2010). Brain-derived neurotrophic factor is associated with age-related decline in hippocampal volume. J Neurosci 30: 5368-5375.

Frisoni GB, Jack CR Jr., Bocchetta M, Bauer C, Frederiksen KS, Liu $\mathrm{Y}$ et al (2015). The EADC-ADNI harmonized protocol for manual hippocampal segmentation on magnetic resonance: evidence of validity. Alzheimers Dement 11: 111-125.

Frodl T, Jager M, Smajstrlova I, Born C, Bottlender R, Palladino T et al (2008). Effect of hippocampal and amygdala volumes on clinical outcomes in major depression: a 3-year prospective magnetic resonance imaging study. J Psychiatry Neurosci 33: 423-430.

Hesse GW, Teyler TJ (1976). Reversible loss of hippocampal long term potentiation following electronconvulsive seizures. Nature 264: 562-564.

Jack CR Jr., Twomey CK, Zinsmeister AR, Sharbrough FW, Petersen RC, Cascino GD (1989). Anterior temporal lobes and hippocampal formations: normative volumetric measurements from MR images in young adults. Radiology 172: 549-554.

Jain S, Sima DM, Ribbens A, Cambron M, Maertens A, Van Hecke W et al (2015). Automatic segmentation and volumetry of multiple sclerosis brain lesions from MR images. Neuroimage Clin 8: 367-375.

Jorgensen A, Magnusson P, Hanson LG, Kirkegaard T, Benveniste H, Lee $\mathrm{H}$ et al (2015). Regional brain volumes, diffusivity, and metabolite changes after electroconvulsive therapy for severe depression. Acta Psychiatr Scand 133: 154-164.

Joshi SH, Espinoza RT, Pirnia T, Shi J, Wang Y, Ayers B et al (2015). Structural plasticity of the hippocampus and amygdala induced by electroconvulsive therapy in major depression. Biol Psychiatry 79: 282-292.

Kerner N, Prudic J (2014). Current electroconvulsive therapy practice and research in the geriatric population. Neuropsychiatry (London) 4: 33-54.

Kunigiri G, Jayakumar PN, Janakiramaiah N, Gangadhar BN (2007). MRI T(2) relaxometry of brain regions and cognitive dysfunction following electroconvulsive therapy. Indian $J$ Psychiatry 49: 195-199. 
Molendijk ML, Spinhoven P, Polak M, Bus BA, Penninx BW, Elzinga BM (2013). Serum BDNF concentrations as peripheral manifestations of depression: evidence from a systematic review and metaanalyses on 179 associations $(\mathrm{N}=9484)$. Mol Psychiatry 19: 791-800.

Montgomery SA, Asberg M (1979). A new depression scale designed to be sensitive to change. Br J Psychiatry 134: 382-389.

Nickl-Jockschat T, Palomero GN, Kumar V, Hoffstaedter F, Brugmann E, Habel U et al (2016). Are morphological changes necessary to mediate the therapeutic effects of electroconvulsive therapy? Eur Arch Psychiatry Clin Neurosci 266: 261-267.

Nordanskog P, Dahlstrand U, Larsson MR, Larsson EM, Knutsson L, Johanson A (2010). Increase in hippocampal volume after electroconvulsive therapy in patients with depression: a volumetric magnetic resonance imaging study. J ECT 26: 62-67.

Nordanskog P, Larsson MR, Larsson EM, Johanson A (2014). Hippocampal volume in relation to clinical and cognitive outcome after electroconvulsive therapy in depression. Acta Psychiatr Scand 129: 303-311.

Nordgren M, Karlsson T, Svensson M, Koczy J, Josephson A, Olson L et al (2013). Orchestrated regulation of nogo receptors, lotus, AMPA receptors and BDNF in an ECT model suggests opening and closure of a window of synaptic plasticity. PLoS One 8: e78778.

Ota M, Noda T, Sato N, Okazaki M, Ishikawa M, Hattori K et al (2015). Effect of electroconvulsive therapy on gray matter volume in major depressive disorder. J Affect Disord 186: 186-191.

Pilar-Cuellar F, Vidal R, Diaz A, Castro E, dos Anjos S, Pascual-Brazo J et al (2013). Neural plasticity and proliferation in the generation of antidepressant effects: hippocampal implication. Neural Plast, (2013)537265.

Polyakova M, Schroeter ML, Elzinga BM, Holiga S, Schoenknecht P, de Kloet ER et al (2015). Brain-derived neurotrophic factor and antidepressive effect of electroconvulsive therapy: systematic review and meta-analyses of the preclinical and clinical literature. PLoS One 10: e0141564.

Price JL, Drevets WC (2010). Neurocircuitry of mood disorders. Neuropsychopharmacology 35: 192-216.

Rapinesi C, Kotzalidis GD, Curto M, Serata D, Ferri VR, Scatena P et al (2015). Electroconvulsive therapy improves clinical manifestations of treatment-resistant depression without changing serum BDNF levels. Psychiatry Res 227: 171-178.
Sartorius A, Demirakca T, Böhringer A, von Hogenberg C, Aksay SS, Bumb JM et al (2016). Electroconvulsive therapy increases temporal gray matter volume and cortical thickness. Eur Neuropsychopharmacol 26: 506-517.

Sartorius A, Hellweg R, Litzke J, Vogt M, Dormann C, Vollmayr B et al (2009). Correlations and discrepancies between serum and brain tissue levels of neurotrophins after electroconvulsive treatment in rats. Pharmacopsychiatry 42: 270-276.

Sen S, Duman R, Sanacora G (2008). Serum brain-derived neurotrophic factor, depression, and antidepressant medications: meta-analyses and implications. Biol Psychiatry 64: 527-532.

Sexton CE, Mackay CE, Ebmeier KP (2013). A systematic review and meta-analysis of magnetic resonance imaging studies in latelife depression. Am J Geriatr Psychiatry 21: 184-195.

Sheehan DV, Lecrubier Y, Sheehan KH, Amorim P, Janavs J, Weiller E et al (1998). The Mini-International Neuropsychiatric Interview (M.I.N.I.): the development and validation of a structured diagnostic psychiatric interview for DSM-IV and ICD-10. J Clin Psychiatry 59(Suppl 20): 22-33.

Sousa N, Lukoyanov NV, Madeira MD, Almeida OF, Paula-Barbosa MM (2000). Reorganization of the morphology of hippocampal neurites and synapses after stress-induced damage correlates with behavioral improvement. Neuroscience 97: 253-266.

Spalding KL, Bergmann O, Alkass K, Bernard S, Salehpour M, Huttner HB et al (2013). Dynamics of hippocampal neurogenesis in adult humans. Cell 153: 1219-1227.

Szabo K, Hirsch JG, Krause M, Ende G, Henn FA, Sartorius A et al (2007). Diffusion weighted MRI in the early phase after electroconvulsive therapy. Neurol Res 29: 256-259.

Tendolkar I, van Beek M, van Oostrom I, Mulder M, Janzing J, Oude Voshaar R et al (2013). Electroconvulsive therapy increases hippocampal and amygdala volume in therapy refractory depression: a longitudinal pilot study. Psychiatry Res 214: 197-203.

Vaidya VA, Siuciak JA, Du F, Duman RS (1999). Hippocampal mossy fiber sprouting induced by chronic electroconvulsive seizures. Neuroscience 89: 157-166.

van der Lijn F, den Heijer T, Breteler MM, Niessen WJ (2008). Hippocampus segmentation in MR images using atlas registration, voxel classification, and graph cuts. Neuroimage 43: $708-720$. 